\title{
EVOLUÇÃO DA COMPLETUDE DAS INFORMAÇÕES SOBRE MORTALIDADE NEONATAL EM MINAS GERAIS
}

\section{EVOLUTION OF THE COMPLETENESS OF INFORMATION ON NEONATAL MORTALITY IN MINAS GERAIS}

\section{EVOLUCIÓN DE LA COMPLETUD DE LAS INFORMACIONES SOBRE MORTALIDAD NEONATAL EN MINAS GENERALES}

\author{
Verônica Aparecida Ferreira ${ }^{1}$, Damiana Aparecida Trindade Monteiro ${ }^{2}$, Luan Augusto Alves \\ Garcia $^{3}$, Fernanda Carolina Camargo ${ }^{4}$, Divanice Contim ${ }^{5}$, Jesislei Bonolo do Amaral ${ }^{6}$
}

\begin{abstract}
RESUMO
Objetivo: analisar a evolução temporal e a qualidade das informações sobre a mortalidade neonatal em Minas Gerais. Métodos: estudo ecológico e de tendência temporal, utilizando informações relacionadas as Declaração de Óbito neonatal disponibilizadas em sistema eletrônico de domínio público no período de 1996 a 2014. Foram considerados aspectos maternos e da gestação, características do parto, do recém-nascido e aspectos relacionados ao óbito neonatal para a classificação da completude e, definição do modelo polinomial para avaliação da tendência por coeficiente de determinação e correlação de Pearson. Resultados: variáveis sexo do recém-nascido (média=0,6; IC 95\% 0,5-0,7), idade do óbito (média=0,3;IC $95 \%$ 0,1-0,5) e local do óbito (média=0,3;IC 95\% 0,1-0,4) apresentaram excelente completude. Modelo polinomial de primeira ordem foi determinante para explicação de tendência de redução da incompletude na maioria das variáveis (82\%). Conclusão: apesar da tendência de redução da incompletude, é relevante fomentar a discussão sobre o tema, tendo em vista a melhor vigilância dos óbitos neonatais.
\end{abstract}

Descritores: Mortalidade infantil; Sistemas de Informação em Saúde; Recém-nascido.

\footnotetext{
${ }^{1}$ Enfermeira. Especialista em Enfermagem em Neonatologia. Enfermeira da Estratégia Saúde da Família do município de Uberaba (MG), Brasil.

${ }^{2}$ Mestre em Atenção à Saúde. Doutoranda em Atenção à Saúde pela Universidade Federal do Triângulo Mineiro (UFTM), Uberaba/MG.

${ }^{3}$ Enfermeiro. Especialista em Enfermagem do Trabalho, Gestão dos Serviços em Ergonomia, Gestão Pública em Saúde pela Faculdade de Gestão e Negócios da Universidade Federal de Uberlândia (UFU), Saúde do Adulto na modalidade Residência Multiprofissional em Saúde pela Universidade Federal do Triângulo Mineiro - UFTM.

${ }^{4}$ Mestre e Doutora do Programa de Pós-Graduação Stricto Sensu em Atenção à Saúde da UFTM. Epidemiologista Clínica do Setor de Pesquisa e Inovação Tecnológica da Gerencia de Ensino e Pesquisa - Hospital de Clínicas da Universidade Federal do Triângulo Mineiro (UFTM) - Uberaba (MG), Brasil.

${ }^{5}$ Enfermeira. Mestre e Doutora pela Universidade Federal de São Paulo. Professora Adjunta do Curso de Graduação em Enfermagem da Universidade Federal do Triângulo Mineiro (UFTM) - Uberaba (MG), Brasil.

${ }^{6}$ Enfermeira. Doutora em Atenção à Saúde da UFTM. Professora Adjunta do Curso de Graduação em Enfermagem da Universidade Federal do Triângulo Mineiro (UFTM) - Uberaba (MG), Brasil.
} 


\begin{abstract}
Objective: To analyze the evolution and the quality of information on neonatal mortality in Minas Gerais. Method: ecological study of time trends and using information related to dealth certificates neonatal available in electronic system of public domain in the period from 1996 to 2014. They considered aspects maternal and pregnancy, the birth and the newborn characteristics and aspects related to neonatal death for the completeness classification and definition of the polynomial model to evaluate the trend for coefficient of determination and Pearson correlation. Results: Variables sex of the newborn (mean $=0.6$, CI95\% 0.5-0.7), age at death (mean $=0.3$, CI95\% 0.1-0.5) and local of death (mean $=0.3$, CI95\% 0.1-0.4) showed excellent completeness. polynomial model of the first order was crucial to explaining incompleteness downward trend in most of the variables (82\%). Conclusion: Despite the incompleteness downward trend, it is important to stimulate discussion on the issue, with a view to better monitoring of neonatal deaths.
\end{abstract}

Descriptors: Infant Mortality; Health Information Systems; Newborn Infant.

\title{
RESUMEN
}

Objetivo: Analizar la evolución temporal y la calidad de las informaciones sobre la mortalidad neonatal en Minas Gerais. Métodos: Estudio ecológico y de tendencia temporal, utilizando informaciones relacionadas a la Declaración de Óbito neonatal disponibilizadas en sistema electrónico de dominio público en el período de 1996 a 2014. Se consideraron aspectos maternos y de gestación, características del parto, del recién nacido y aspectos relacionados al óbito neonatal para la clasificación de la completitud y, definición del modelo polinomial para la evaluación de la tendencia por coeficiente de determinación y correlación de Pearson. Resultados: Variables sexo: del recién nacido (promedio $=0,6$, IC 95\% 0,5-0,7), edad del óbito (media $=0,3$, IC 95\% 0,1-0,5) y lugar de la muerte $=0,3$, IC 95\% 0,1-0,4) presentaron excelente completitud. El modelo polinomial de primer orden fue determinante para explicar la tendencia de reducción de la incompletud en la mayoría de las variables (82\%). Conclusión: A pesar de la tendencia de reducción de la incompletud, es relevante fomentar la discusión sobre el tema, con vistas a la mejor vigilancia las muertes neonatales.

Descriptores: Mortalidad Infantil; Sistemas de Información en Salud; Recién Nacido.

\section{INTRODUÇÃO}

A mortalidade infantil consiste na morte de crianças no primeiro ano de vida, reconhecida como um indicador sensível das condições de vida e saúde de uma população. ${ }^{1}$ A mortalidade neonatal (0 a 27 dias de vida) é o principal componente da mortalidade infantil (ONU, 2015), sendo que em 2016 ocorreram 20.176 óbitos no período neonatal precoce no Brasil. ${ }^{2}$

Informações referentes ao óbito neonatal podem ser obtidas por meio do
Sistema de Informação de Mortalidade (SIM), disponível pela internet de bastante acessível. ${ }^{3}$ Esta ferramenta permite maior facilidade para o monitoramento da mortalidade neonatal, entretanto é preciso cada vez mais investir em estudos que utilizem esse Sistema de Informação em Saúde (SIS) como base de dados, sendo que a literatura atual aponta para fragilidades importantes quanto à qualidade das informações contidas nessas bases. ${ }^{2-3}$ 
Estudos sobre os fatores de risco envolvidos na cadeia causal da mortalidade neonatal e o reconhecimento do comportamento dos óbitos ao longo do tempo são fundamentais para a definição de ações prioritárias apropriadas para contenção desses óbitos. Com isso, ter SIS qualificados quanto a completude e abrangência sobre as estatísticas vitais, de fácil acesso, promove maior viabilidade para a efetivação de ações de vigilância desses óbitos. ${ }^{3}$

A Organização das Nações Unidas ONU ao analisar os maiores problemas mundiais, estabeleceu oito Objetivos de Desenvolvimento do Milênio, sendo o oitavo a redução da mortalidade infantil, que visa reduzir em 2/3 a taxa global de mortalidade infantil. ${ }^{1}$ Entre as iniciativas para a redução da mortalidade infantil é importante promover o registro correto da Declaração de Óbito (DO), bem como melhorar o registro da causa básica, fortalecendo os sistemas nacionais de informação sanitária, permitindo melhoria nas estimativas futuras da mortalidade infantil. $^{3,4}$ Desta maneira, revela-se a importância da compreensão das lacunas existentes para a efetivação da qualidade das informações disponíveis nos SIS, tendo em vista o impacto que reflete para o alcance das metas do milênio.

Neste contexto, a redução dos óbitos neonatais é um desafio quando relacionado as desigualdades socioeconômicas existentes nos países em desenvolvimento. ${ }^{4}$ Sendo que essas desigualdades acabam por impactar na qualidade dos registros sobre as estatísticas vitais do neonato. Por conseguinte, aspecto relevante para o desenvolvimento da presente pesquisa relaciona-se a pouca literatura existente sobre este fenômeno em territórios específicos. ${ }^{5-6}$

Com relação a estudos epidemiológicos que utilizam dados secundários é inegável a sua contribuição. Todavia, é preciso destacar algumas limitações, enquanto fonte de dados. O SIM é de abrangência nacional, desenvolvido pelo Departamento de Informática do Sistema Único de Saúde, pelo qual são consolidadas todas as DOs. Esses registros são considerados as fontes mais ágeis na enumeração e quantificação dos óbitos, no Brasil. $^{7}$

Observa-se na literatura escassez de estudos relacionados à avaliação da qualidade da informação para a mortalidade neonatal no Brasil. Frente a essa realidade, o presente estudo questiona: Qual a completude das informações relacionadas ao óbito neonatal em Minas Gerais? A qualidade desta completude melhora com o passar dos anos? Para tanto, objetiva-se analisar a evolução temporal e a completude das informações sobre a mortalidade neonatal no estado de Minas Gerais. 


\section{MÉTODO}

Trata-se de estudo epidemiológico, ecológico e de tendência temporal. Foram utilizadas informações disponíveis no SIM, referentes à Classificação Internacional de Doenças em sua décima revisão (CID-10). Foram incluídos todos os registros de óbitos neonatal, para o período de 1996 a 2014, de mães residentes em Minas Gerais. O período proposto apresentou-se pela disponibilidade das informações no sítio eletrônico no período da coleta.

Todas as informações foram consideradas, incluindo aquelas que apresentaram campo em branco ou ignorado, por ser variável de interesse para a análise da completude dos registros. A extração dos dados ocorreu entre 25 de maio a 17 de junho de 2016, foi feita por pares de forma independente e depois conferida e ajustadas inadequações.

As variáveis de estudo foram compostas por: aspectos maternos e da gestação (idade materna; escolaridade materna; duração gestacional; tipo de gravidez), características do parto e do $\mathrm{RN}$ (tipo de parto; peso ao nascer; sexo; cor da pele/raça) e aspectos relacionados ao óbito neonatal (idade do óbito do neonato; local de ocorrência do óbito; causas do óbito), de acordo com as classificações disponíveis no SIM/DATASUS. Destaca-se que para análise da completude das causas do óbito, adaptou-se o campo para a proporção de causas óbito mal definidas.

A análise da completude baseou-se nos critérios propostos pela Comissão Econômica para a América Latina e o Caribe (Cepal), que considera a proporção de informação ignorada, os campos em branco e os códigos atribuídos como informação ignorada especificada, caracterizados como incompletude de informação. ${ }^{8}$ Foi realizada uma proporção entre o total de variáveis analisadas e as variáveis que se encontram preenchidas como ignorada ou campos em branco, conforme a seguinte expressão: $n^{\circ} d e$ variáveis em branco ou ignoradas $/ n^{\circ}$ total de variáveis $x 100$.

Apresentou-se análise exploratória descritiva do comportamento desta proporção ao longo dos anos por frequência absoluta, média (para o período de 19 anos) e Intervalo de confiança de (IC95\%). Estes dados foram apresentados conforme média trienal (1996/1998, 1999/2001, 2002/2004, 2005/2007, 2008/2010) e uma média quadrienal (2011/2014).

A avaliação da incompletude seguiu as considerações da Cepal que aborda os seguintes padrões: excelentes quando há menos de 5\% de informações incompletas, bons de $5 \%$ a $10 \%$, regulares de $10 \%$ a $20 \%$, ruins de $20 \%$ a $50 \%$ e muito ruins de $50 \%$ ou mais. ${ }^{8}$ 
Para a análise da tendência da incompletude das informações nos registros de óbito neonatal, utilizou-se modelo de regressão polinomial de primeira ordem, considerando a proporção de incompletude ( $n^{\circ}$ de variáveis em branco ou ignoradas / $n^{\circ}$ total de variáveis $x$ 100) para a variável dependente $(\mathrm{Y})$ e a variável independente (X) os anos referentes ao período de estudo. Foi utilizada a variável X centralizada (ano menos o ponto médio da série histórica), com o ano de 2005 como ponto médio. Foram construídos diagramas de dispersão entre as proporções e os anos de estudo para identificar a função que expressasse a relação entre eles. A partir desta relação, estimaram-se modelos de regressão polinomial que além da análise do poder estatístico também fossem de fácil interpretação. $^{9}$

A tendência apresentou como medida de precisão o coeficiente de determinação $\left(r^{2}\right)$ e correlação linear de Pearson (r) sendo classificada em correlação nula $(\mathrm{r}=0)$, correlação fraca $(0$ $<\mathrm{r}<0,3)$, correlação regular $(0,3 \leq \mathrm{r}<0,6)$, correlação forte $(0,6 \leq \mathrm{r}<0,9)$, correlação muito forte $(0,9 \leq \mathrm{r}<1)$ e, correlação plena $(\mathrm{r}=1)$, para variações positivas e negativas. Inicialmente testou-se o modelo de regressão linear simples $(Y=\beta 0+\beta 1 \mathrm{X})$. Foi considerada significativa quando o modelo estimado obteve $p \leq 0,05$. Caso a regressão linear simples apresentasse $r^{2} \geq 70 \%$ e $p \leq$
0,05, foi o modelo eleito. Foi identificada a presença de tendência nas séries como crescente ou decrescente conforme sinal determinante de $\beta 1$ (positivo ou negativo) e sentido da reta.

Os dados foram extraídos do sítio eletrônico do SIM/DATASUS em formato de tabela Excel®. Para as análises estatísticas, o banco de dados foi transposto para software Statistical Package for the Social Sciences (SPSS) versão 21.0.

O estudo foi apreciado pelo Comitê de Ética e dispensado de análise por considerar a natureza da investigação e desenho metodológico exclusivo com dados secundários de acesso público pela internet.

\section{RESULTADOS}

Em Minas Gerais, para o período de análise (1996-2014), ocorreram 62.699 óbitos neonatais conforme informações do SIM. Esses óbitos perfizeram 9,5\% do total de óbitos do território nacional para o período, o qual foi de 663.683 óbitos neonatais. Observa-se que a taxa de mortalidade neonatal para Minas Gerais é a terceira taxa na Região Sudeste com valor correspondente a 8,2 óbitos/1000 nascidos vivos para o ano de 2014.

Sobre a completude das informações, apesar de existirem aspectos preenchidos de forma excelente, isto é, com menos campos incompletos como: local do óbito (média =0,3; IC95\% 0,1 - 0,4), idade 
do óbito (média $=0,3 ;$ IC95\% 0,1 - 0,5) e sexo do RN (média = 0,6; IC95\% 0,5 - 0,7), a maior parte das informações apresenta preenchimento com qualidade classificada como ruim e muito ruim (7 campos do total de 11 campos analisados; 63,6\%). O campo que apresenta pior preenchimento é a escolaridade materna, classificado como muito ruim, apresentando proporção de incompletude conforme média para o período de 50,3 (IC95\% 35,6 - 54,9) (Tabela 1). 
Tabela 1. Distribuição da proporção de incompletude das informações nos registros dos óbitos neonatais/SIM conforme média, intervalo de confiança (95\%) e sua classificação para o período 1996 a 2014. Minas Gerais, Brasil, 2016.

\begin{tabular}{|c|c|c|c|c|c|c|c|c|c|}
\hline Variáveis & $1996 / 1998$ & $1999 / 2001$ & $2002 / 2004$ & $2005 / 2007$ & $2008 / 2010$ & $2011 / 2014$ & Média & IC95\% & Completude dos campos $^{\mathrm{a}}$ \\
\hline \multicolumn{10}{|c|}{ Aspectos maternos e da Gestação } \\
\hline Idade materna & 37,0 & 44,9 & 36,9 & 23,9 & 25,4 & 17,5 & 32,5 & $25,4-35,1$ & Ruim \\
\hline Escolaridade materna & 84,3 & 54,4 & 46,2 & 34,0 & 34,1 & 25,2 & 50,3 & $35,6-54,9$ & Muito Ruim \\
\hline Duração gestacional & 47,8 & 39,9 & 29,2 & 18,1 & 15,5 & 19,3 & 31,0 & $21,8-33,9$ & Ruim \\
\hline Tipo de gravidez & 44,7 & 36,8 & 26,7 & 16,7 & 14,5 & 12,6 & 27,9 & $18,6-30,7$ & Ruim \\
\hline \multicolumn{10}{|c|}{ Características do Parto e do Recém-Nascido } \\
\hline Tipo de parto & 42,8 & 37,3 & 27,4 & 17,5 & 15,4 & 13,1 & 28,1 & $19,2-30,7$ & Ruim \\
\hline Peso ao nascer & 49,2 & 41,5 & 30,8 & 18,5 & 16,3 & 13,4 & 31,2 & $20,7-34,5$ & Ruim \\
\hline Sexo & 0,5 & 0,7 & 0,5 & 0,5 & 0,5 & 0,8 & 0,6 & $0,5-0,7$ & Excelente \\
\hline Cor da pele & 69,4 & 40,0 & 34,5 & 25,2 & 17,8 & 15,0 & 37,6 & $22,5-42,8$ & Ruim \\
\hline \multicolumn{10}{|c|}{ Aspectos Relacionados ao óbito } \\
\hline Idade do óbito & 0,2 & 0,2 & 1,0 & 0,5 & 0,1 & 0,0 & 0,3 & $0,1-0,5$ & Excelente \\
\hline Causas do óbito* & 18,8 & 19,4 & 15,1 & 7,0 & 2,9 & 1,7 & 12,3 & $6,6-14,1$ & Regular \\
\hline Local do óbito & 0,5 & 0,6 & 0,1 & 0,1 & 0,1 & 0,1 & 0,3 & $0,1-0,4$ & Excelente \\
\hline
\end{tabular}

*Considerada a proporção de Causas Mal definidas

${ }^{a}$ Classificação Cepal tendo a média como referência 
Em relação à tendência da completude dos campos com a evolução temporal, observa-se que o modelo polinomial de primeira ordem (linear) representa esta relação para a maior parte das variáveis analisadas, frente aos resultados do coeficiente de determinação $\left(r^{2} \geq 70 \%\right)$, exceto para as variáveis: local de ocorrência do óbito, idade do óbito e sexo do $\mathrm{RN}\left(\mathrm{r}^{2}=55 \%, 10 \%\right.$ e $4 \%$, respectivos). Quanto à correlação (r) entre qualidade do preenchimento e evolução temporal encontra-se forte ou muito forte em $82 \%$ das variáveis (Tabela 2).

Observa-se que $91 \%$ das variáveis comportam-se conforme modelo de forma decrescente frente à evolução temporal, com significância estatística. Apenas a variável sexo do $\mathrm{RN}$ não apresentou melhoria na qualidade de preenchimento com o passar dos anos, sendo a tendência crescente. Entretanto, esta variável apresenta excelente qualidade de preenchimento, e o modelo polinomial de primeira ordem apresenta fraca capacidade explicativa da tendência desta variável $\left(\mathrm{r}^{2}=\right.$ $4 \%, \quad p=0,43)$. Outra variável com comportamento similar na análise da regressão polinomial de primeira ordem foi à idade do óbito que apresentou qualidade de preenchimento excelente, porém tendência decrescente $\left(\mathrm{r}^{2}=10 \%, p=0,19\right)$ (Tabela 2).

Tabela 2. Modelo de regressão da análise de tendência da proporção de incompletude dos campos da Declaração de óbito neonatal/SIM segundo aspectos maternos, gestacionais, do parto, do recém-nascido e relacionados ao óbito, para o período 1996 a 2014. Minas Gerais, Brasil, 2016.

\begin{tabular}{|c|c|c|c|c|c|}
\hline Variáveis & Modelo $^{\mathrm{a}}$ & $r^{2}$ & valor $p$ & Classificação (r) & Tendência \\
\hline \multicolumn{6}{|c|}{ Aspectos Maternos e da Gestação } \\
\hline Idade materna & $y=-1,586 x+3210$ & $78 \%$ & $<0,001$ & Forte $(-0,8)$ & Decrescente \\
\hline Escolaridade materna & $y=-3,306 x+6674$ & $85 \%$ & $<0,001$ & Muito Forte $(-0,9)$ & Decrescente \\
\hline Duração da Gestação & $y=-2,009 x+4056$ & $80 \%$ & $<0,001$ & Muito Forte $(-0,9)$ & Decrescente \\
\hline Tipo de gravidez & $y=-2,125 x+4286$ & $90 \%$ & $<0,001$ & Muito Forte $(-0,9)$ & Decrescente \\
\hline \multicolumn{6}{|c|}{ Características do Parto e do Recém-Nascido } \\
\hline Tipo de parto & $y=-2,033 x+4102$ & $91 \%$ & $<0,001$ & Muito Forte $(-0,9)$ & Decrescente \\
\hline Peso ao nascer & $y=-2,414 x+4868$ & $92 \%$ & $<0,001$ & Muito Forte $(-0,9)$ & Decrescente \\
\hline Sexo & $y=0,008 x-17,10$ & $4 \%$ & 0,43 & Fraca $(0,2)$ & Crescente \\
\hline Cor/Raça & $y=-3,175 x+6399$ & $72 \%$ & $<0,001$ & Forte $(-0,8)$ & Decrescente \\
\hline \multicolumn{6}{|c|}{ Aspectos Relacionados ao óbito } \\
\hline Idade do óbito & $y=-0,023 x+47,65$ & $10 \%$ & 0,19 & Regular $(-0,3)$ & Decrescente \\
\hline Causas do óbito* & $y=-1,299 x+2614$ & $89 \%$ & $<0,001$ & Muito Forte $(-0,9)$ & Decrescente \\
\hline $\begin{array}{l}\text { Local de ocorrência do } \\
\text { óbito }\end{array}$ & $y=-0,033 x+68,06$ & $55 \%$ & $<0,001$ & Forte $(-0,7)$ & Decrescente \\
\hline
\end{tabular}




\section{DISCUSSÃO}

Os resultados da presente pesquisa apontam para uma variação na completude das informações estudadas. As variáveis referentes ao sexo do RN, à idade do óbito e ao local do óbito apresentaram qualidade de preenchimento excelente. Essas variáveis também apresentam excelência na completude em outros estados brasileiros, como Pernambuco ${ }^{10,11}$ e Espírito Santo. ${ }^{12}$

Em âmbito nacional, outras variáveis, como as socioeconômicas e demográficas, também apresentam baixa qualidade no preenchimento. A exemplo, a escolaridade materna, uma das variáveis mais relevantes nos estudos de desigualdade social, apresenta omissão no preenchimento da DO em mais de $50 \%$ de 11 estados brasileiros. $^{8}$ Alerta-se para a qualidade das informações das outras variáveis estudadas nesta pesquisa, que se encontram classificadas como ruim ou muito ruim, e a causa óbito como regular, o quão isto pode impactar nos indicadores de avaliação das condições de saúde. ${ }^{13}$

O desafio em alcançar a qualidade das informações sobre mortalidade dos menores de um ano não é uma questão específica do Brasil, se impondo para diferentes países, em especial ao conjunto considerado em desenvolvimento. ${ }^{14}$ Problemas semelhantes foram encontrados em Cabinda, Angola. Nesta localidade é grande o percentual de subnotificação dos nascimentos e dos óbitos, fato que se associa a má qualidade da assistência à saúde local. ${ }^{15}$

Em Belize, país da América Central, estudo que analisa a integração do sistema de informação sobre mortalidade em 2007 encontrou diversos desafios para o alcance da qualidade, dentre eles a ampliação da integração das informações por toda extensão territorial e a capacitação dos profissionais de saúde em estarem utilizando e alimentando esse sistema de forma adequada. ${ }^{16}$

Entre os fatores referentes à manutenção da qualidade dos dados sobre a mortalidade, ganha destaque o fato de que os médicos preenchem os formulários da DO apenas para cumprir uma formalidade, sem preocupação com o valor da informação. Em geral, estes profissionais ao preencherem a DO - atribuição exclusiva desta categoria, demonstram maior preocupação em informar a causa imediatamente associada ao óbito e não os outros fatores determinantes. Neste contexto, é fundamental a sensibilização da classe para o impacto na completude dos registros. ${ }^{11}$

Sobre o preenchimento da DO em caso de morte neonatal, pesquisa evidenciou que mesmo em óbitos ocorridos no hospital, os quais apresentam maior possibilidade de resgate de informações para preenchimento da DO pela 
proximidade da família e do prontuário, o percentual de incompletude dos campos é muito elevado. ${ }^{11}$

Doravante, o elevado percentual de variáveis ignoradas ou em branco na DO podem alterar o perfil da mortalidade infantil, haja vista que a busca ativa de óbitos não informados ao SIM pode constatar essas modificações, inclusive na causa básica do óbito. ${ }^{10}$ Sobretudo, o preenchimento deficitário dos campos da DO compromete a qualidade e a pertinência das informações disponíveis, transformando-se em obstáculo para a adoção adequada da vigilância epidemiológica. ${ }^{11}$

Outro motivo para a incompletude das informações relaciona-se a fragilidades encontradas na operacionalização do SIM. Este fato, conforme apontam estudos, sinaliza a necessidade de mudanças no processo de trabalho, desde o preenchimento, alimentação do sistema, ambientação amigável do sistema e até mesmo na instauração de processo de auditoria interna das informações. ${ }^{10,16}$

O Brasil avançou na redução da mortalidade infantil, estando no caminho para atingir a quarta meta do milênio, caso mantenha a tendência de decréscimo desses óbitos. ${ }^{4}$ Entretanto, é preciso localmente avançar na qualidade dos registros, já que é imprescindível investimentos para ampliar a qualidade da completude dos registros dos óbitos neonatais. ${ }^{17}$

Historicamente, investimentos a fim de incrementar a qualidade das informações se deram no Brasil recentemente, pelo grande apoio acadêmico e pela adoção de recomendações da Organização Mundial de Saúde para que se procedesse à revisão e consolidação dos sistemas de informação em saúde. ${ }^{17}$ De forma geral, é possível identificar uma tendência positiva para a completude das informações ao se avaliar o SIM em âmbito nacional, relacionadas ao óbito neonatal ${ }^{8,10,16-17}$, sendo que o estado de Minas Gerais acompanha essa tendência conforme aponta os resultados desta pesquisa.

O estado de Minas Gerais apresenta diversos desafios para o alcance da qualidade das informações relacionadas ao óbito neonatal ${ }^{18}$, realidade confirmada também pelos resultados desta pesquisa. Sendo um estado de desigualdades sócio regionais em aspectos econômicos e de acesso e qualidade dos serviços de saúde que precisam ser consideradas. ${ }^{19}$

Por sua vez, são necessários investimentos e políticas públicas afirmativas para o aperfeiçoamento da vigilância do óbito neonatal, integração dos comitês de prevenção do óbito materno, fetal e infantil além de capacitação de todos os envolvidos na produção da informação e busca ativa de eventos. ${ }^{17,20}$ Estes são 
exemplos de intervenções estratégicas para contribuir na redução das inadequações das informações sobre mortalidade infantil no território de Minas Gerais e, portanto, em outras localidades que apresentem problemas similares.

\section{CONCLUSÃO}

Os dados apontam tendência positiva para a melhoria da qualidade no preenchimento das informações obtidas no SIM sobre o óbito neonatal, quanto a sua evolução temporal. Entretanto, a maior parte das variáveis estudadas encontra-se com qualidade de completude ruim ou muito ruim e a causa óbito com qualidade regular de preenchimento.

Quanto as limitações deste estudo, há de se considerar a qualidade das informações do SIM, que embora possa apresentar subregistros de informações, ainda é uma importante fonte de informações em nosso país. Outra questão se relacioana a utilização de dados agregados, quais não permitem análise de riscos individuais.

Tendo em vista a extensão territorial de Minas Gerais e as desigualdades sociais presentes nas suas diferentes regiões, é importante que pesquisas sejam realizadas para a compreensão do impacto das iniquidades de saúde na qualidade das informações. Além de reconhecer quais fatores determinam a baixa completude dos campos e influenciam a tendência positiva com o passar dos anos. Delineamentos qualitativos de pesquisas junto aos médicos tornam-se relevantes para compreensão das resistências ao preenchimento adequado da DO sobre óbitos neonatais. Como também, estudos para a correção dos sub-registros e para avaliação do impacto de iniciativas para melhorar a qualidade das informações do SIM.

\section{REFERÊNCIAS}

1. Organização das Nações Unidas.

Relatório sobre os objetivos de desenvolvimento do Milênio [Internet]. 2015 [citado em 09 mai 2019]. Disponível em: http://abm.org.br/ods/wpcontent/uploads/2017/10/Relatorio-sobreos-Objetivos-do-Milenio-2015.pdf

2. Ministério da Saúde (Brasil), DATASUS - Departamento de Informática do SUS. Estatísticas vitais [Internet].

Brasília, DF: Ministério da Saúde; 2018 [citado em 14 maio 2019]. Disponível em: http://www.datasus.gov.br/DATASUS/ind ex.php?area $=0205$

3. Feitosa AC, Santos EFS, Ramos JLS, Bezerra IMP, Nascimento VG, Macedo $\mathrm{CC}$, et al. Factors associated with infant mortality in the metropolitan region of Cariri, Ceará, Brazil. Rev Bras Crescimento Desenvolv Hum. [Internet]. 2015 [citado em 14 maio 2019]; 25(2):22429. doi: http://dx.doi.org/10.7322/jhgd.103019 4. Vieira LB, Gouveia HG, Wegner W, Gerhardt LM. The millennium development goals and the social commitment of nursing research. Rev Gaúch Enferm. [Internet]. 2015 [citado em 14 maio 2019]; 36(1):8-9. doi: http://dx.doi.org/10.1590/19831447.2015.01.53436 
5. Dutra IR, Andrade GN, Rezende EM, Gazzinelli A. Investigação dos óbitos infantil e fetal no Vale do Jequitinhonha, Minas Gerais, Brasil. REME Rev Min Enferm. [Internet]. 2015 [citado em 14 maio 2019]; 19(3):597-604. doi: http://dx.doi.org/ 10.5935/14152762.20150046

6. Pinheiro JMF, Tinoco LS, Rocha ASS, Rodrigues MP, Lyra CO, Ferreira MAF. Atenção à criança no período neonatal: avaliação do pacto de redução da mortalidade neonatal no Rio Grande do Norte, Brasil. Ciênc Saúde Colet. [Internet]. 2016 [citado em 13 maio 2019]; 21(1):243-52. doi: http://dx.doi.org/10.1590/141381232015211.09912014

7. Silva LP, Moreira CMM, Amorim MHC, Castro DS, Zandonade E. Avaliação da qualidade dos dados do Sistema de Informações sobre Nascidos Vivos e do Sistema de Informações sobre Mortalidade no período neonatal, Espírito Santo, Brasil, de 2007 a 2009. Ciênc Saúde Colet.

[Internet]. 2014 [citado em 13 maio 2019]; 19(7): 2011-20. doi:

http://dx.doi.org/10.1590/1413-

81232014197.08922013

8. Romero DE, Cunha CB. Avaliação da qualidade das variáveis epidemiológicas e demográficas do Sistema de Informação de Nascidos Vivos, 2002. Cad Saúde Pública [Internet]. 2007 [citado em 13 maio 2019]; 23(3):701-14. doi:

http://dx.doi.org/10.1590/S0102311X2007000300028

9. Antunes JLF, Cardoso MRA. Uso da análise de séries temporais em estudos epidemiológicos. Epidemiol Serv Saúde [Internet]. 2015 [citado em 13 maio 2019]; 24(3):565-76. Disponível em: http://www.scielo.br/pdf/ress/v24n3/22379622-ress-24-03-00565.pdf

10. Figueiroa BQ, Vanderlei LCM, Frias PG, Carvalho PI, Szwarcwald CL. Análise da cobertura do Sistema de Informações sobre Mortalidade em Olinda, Pernambuco, Brasil. Cad Saúde Pública [Internet]. 2013 [citado em 13 maio 2019];
29(3):475-84. doi: http://dx.doi.org/10.1590/S0102-

311X2013000300006

11. Ramalho MOA, Frias PG, Vanderlei

LCM, Macêdo VC, Lira PIC. Avaliação da incompletude da declaração de óbitos de menores de um ano em Pernambuco, Brasil, 1999 a 2011. Ciênc Saúde Colet. [Internet]. 2015 [citado em 14 maio 2019]; 20(9):2891-98. doi: http://dx.doi.org/10.1590/141381232015209.09492014

12. Ribeiro NM, Castro SS, Scatena LM, Haas VJ. Análise da tendência temporal do suicídio e de sistemas de informações em saúde em relação às tentativas de suicídio. Texto \& Contexto Enferm. [Internet]. 2018 [citado em 13 maio 2019];

27(2):e2110016. doi:

http://dx.doi.org/10.1590/0104070720180002110016 14. Mathers CD, Fat DM, Inoue M, Rao C, Lopez AD. Counting the dead and what they died from: an assessment of the global status of cause of death data. Bull World Health Organ. [Internet]. 2005 [citado em 29 jan 2019]; 83(3):171-7. Disponível em: https://apps.who.int/iris/handle/10665/729 66

15. Simão R, Gallo PR. Mortes infantis em Cabinda, Angola: desafios para as políticas públicas de saúde. Rev Bras Epidemiol. [Internet] 2013 [citado em 13 maio 2019]; 16(4):826-37. doi: http://dx.doi.org/10.1590/S1415790X2013000400003

16. Drumond E, Abreu DM, Machado C, Gomes F, Franca E. Racial disparities and avoidable infant mortality in a city of southeastern Brazil, 2001-09. J Trop Pediatr. [Internet]. 2013 [citado em 13 maio 2019]; 59(1):23-8. doi: https://doi.org/10.1093/tropej/fms039 17. Frias PG, Szwarcwald CL, Lira PIC. Avaliação dos sistemas de informações sobre nascidos vivos e óbitos no Brasil na década de 2000. Cad Saúde Pública [Internet] 2014 [citado em 13 maio 2019]; 30(10):2068-80. doi: 
http://dx.doi.org/10.1590/0102-

311X00196113

18. Barbosa TAGS, Coelho KR, Andrade GN, Bittencourt SDA, Leal MC, Gazzinelli

A. Determinantes da mortalidade infantil em municípios do Vale do Jequitinhonha, Minas Gerais, Brasil. REME Rev Min Enferm. [Internet]. 2014 [citado em 13 maio 2019]; 18(4):907-14. doi: http://www.dx.doi.org/10.5935/14152762.20140067

19. Costa GM, Tiburcio JD, Oliveira VC, Gontijo TL, Guimarães EAA.

Determinantes do baixo peso ao nascer a partir das declarações de nascidos vivos. Cienc Enferm. [Internet]. 2014 [citado em 13 maio 2019]; 20(3):21-31. doi: http://dx.doi.org/10.4067/S071795532014000300003 20. AbouZarh C, Rampatige R, Lopez A, de Savigny $\mathrm{D}$. When civil registration is inadequate: interim methods for generating vital statistics. Pac Health Dialog. 2012; 18(1):215-30.

RECEBIDO: $15 / 04 / 19$

APROVADO: 02/09/19

PUBLICADO: 12/2019 